\title{
SPECTRALLY RICH PHASE DISTORTION SOUND SYNTHESIS USING AN ALLPASS FILTER
}

\author{
Joseph Timoney and Victor Lazzarini \\ Sound and Digital Music Technology Research \\ Group \\ NUI Maynooth, \\ Maynooth, Co.Kildare, Ireland \\ joseph.timoney@nuim.ie \\ victor.lazzarinianuim.ie
}

\begin{abstract}
This paper examines a recently introduced technique for sound synthesis that uses a coefficient modulated allpass filter to cause phase modifications to its input signal. The intention in this work is to outline some of the properties of the coefficient modulated allpass filter and then to establish a connection between this new method and the older technique of Phase Distortion. Results are presented to demonstrate how the allpass technique provides a spectrally richer output signal.
\end{abstract}

Index Terms-Acoustic signal processing, audio oscillators, signal synthesis

\section{INTRODUCTION}

Phase Distortion [1] is a synthesis technique that allows the generation of complex spectra by modifying the phase of a sinusoidal signal. This amounts effectively to a subset of phase or frequency modulation (FM) synthesis [2]. The original implementation of Phase Distortion is based on modulating the phase increment used to index a fixed wavetable, not allowing for the use of arbitrary input signals. Recent research [3], [4], [5], [6] and [7] have proposed alternative methods extending FM-related techniques to enable such inputs. Of these, the allpass method [5] seems particularly suited to Phase Distortion applications. It provides a more flexible and generic approach in the implementation of the technique, which can be both employed in synthesis instruments and in digital audio effects units.

In the present article, we first discuss the details of allpass filter coefficient modulation and the derivation of a modulating function from a phase deviation characteristic. This will be followed by a brief introduction to the original Phase Distortion synthesis method. Finally, we present our ideas for an allpass implementation of the technique.

\author{
Jussi Pekonen and Vesa Välimäki \\ Dept. of Signal Processing and Acoustics
TKK Helsinki University of Technology
Espoo, Finland
jussi.pekonen@tkk.fi
vesa.valimaki@tkk.fi

\section{PROPERTIES OF ALLPASS FILTER COEFFICIENT MODULATION}

The transfer function of a time-invariant first-order allpass filter is [5]

$$
H(z)=\frac{-a+z^{-1}}{1-a z^{-1}}
$$

where $a$ is the filter coefficient.

From [8], the phase shift introduced by this filter to its input at a frequency $\omega$ is

$$
\phi(\omega)=-\omega+2 \tan ^{-1}\left(\frac{-a \sin (\omega)}{1-a \cos (\omega)}\right)
$$

If the coefficient is varied over time according to a modulating function $m(t)$ the phase shift will be time-varying and thus will appear as

$$
\phi(\omega)=-\omega+2 \tan ^{-1}\left(\frac{-m(t) \sin (\omega)}{1-m(t) \cos (\omega)}\right)
$$

From eqn. (3), it can be seen that the relationship between the time-varying phase and the modulation function is nonlinear. It is important that the modulating signal is defined between zero and one to avoid dispersive effects associated with allpass filters [5].

To illustrate the relationship given in eqn. (3), consider a modulation signal that is a linear ramp from zero to one, where in [5] it was shown that the filter is stable if $|m(t)| \leq 1$ for all $t$.

The upper panel of Figure 1 shows a plot of the respective phase shift for values of $\omega$ ranging from $261.6 \mathrm{~Hz}$ to $2092.8 \mathrm{~Hz}$ in octave steps, corresponding to the midi notes 60 to 96. In Figure 1's upper panel the non-linear mapping from the modulation to the phase shift can be seen to be more severe at lower frequencies than high frequencies. According to eqn. (3), at a modulation value of 0 the phase shift is $-\omega$ while at a modulation value of 1 the phase shift is $-\pi$. 
By differentiating eqn. (3) we can obtain an equivalent instantaneous frequency deviation to the phase shift imparted by the allpass filter.

$$
\dot{\phi}(\omega, t)=\frac{-2 \dot{m}(t) \sin (\omega)}{1-2 m(t) \cos (\omega)+m^{2}(t)}
$$

where $\dot{m}(t)$ is the time differential of the modulation.
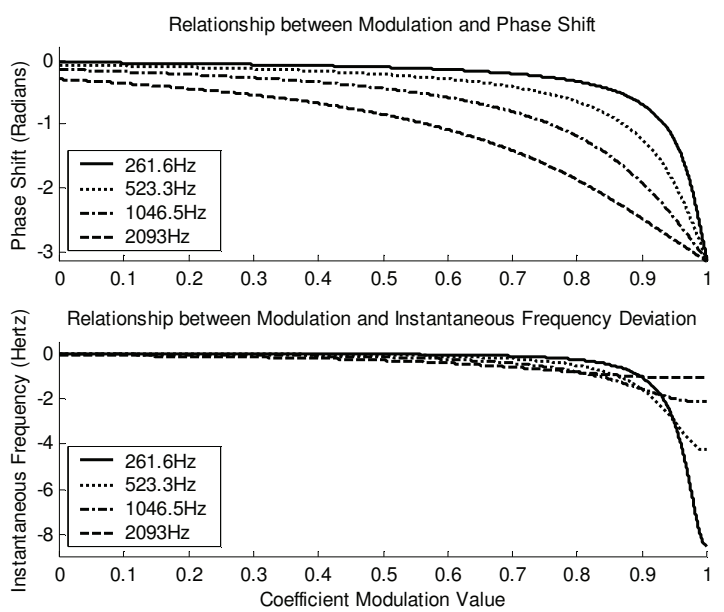

Fig. 1. Relationship between modulation and phase shift at different frequencies (upper panel), and relationship between modulation and instantaneous frequency deviation at different frequencies (lower panel)

Examining eqn. (4) it can be noticed that if the modulation signal is not time-varying, i.e. $\dot{m}(t)=0$, then $\dot{\phi}(\omega, t)=0$. Additionally, the denominator will decrease in magnitude with increasing value of modulation over the range $\omega=0 \ldots \pi / 2$ while the numerator will increase in magnitude with increasing modulation. This behavior is reversed thereafter. The relationship between this instantaneous frequency deviation and the modulation signal can also be investigated graphically. The lower panel of Figure 1 shows a plot of eqn. (4) using the same modulation signal and frequencies used to produce the upper panel. It can be observed in Figure 1's lower panel that only at values of modulation coefficient greater than 0.9 are instantaneous frequency deviations greater than one hertz introduced into the signal. Furthermore, at lower frequencies the deviation introduced is much greater than at higher frequencies. Additionally, the maximum deviation is proportional to the frequency.

\subsection{Obtaining the modulation from the phase deviation}

Assuming we have some desired phase distortion function, for the allpass filter approach it is necessary to find the modulation function that will induce such phase deviations into the filter's input signal.
To determine an expression for the modulation $m(t)$ from the phase deviation $\phi(\omega, t)$ it is possible to rearrange eqn. (3) and following the lead of other authors [9], use a simplifying approximation for the $\tan (x)$ function (for small $x$ ):

$$
\tan \left(\frac{\phi(\omega, t)+\omega}{2}\right) \approx \frac{(\phi(\omega, t)+\omega)}{2}
$$

This will provide an expression for the time-varying modulation function given a time-varying phase shift

$$
m(t)=\frac{-(\phi(\omega, t)+\omega)}{2 \sin (\omega)-(\phi(\omega, t)+\omega) \cos (\omega)}
$$

\section{THE PHASE DISTORTION TECHNIQUE FOR SOUND SYNTHESIS}

The process of Phase Distortion as described in [1] was implemented by modifying the rate at which a one cycle waveform was read out from a wavetable. The change in rate was controlled by a phase distortion function. This approach was the most suitable given the digital hardware used to implement the algorithm. However, it is possible to achieve the same result in software. In [1] the first example of the technique demonstrates how it can be used to generate a sawtooth type waveform.
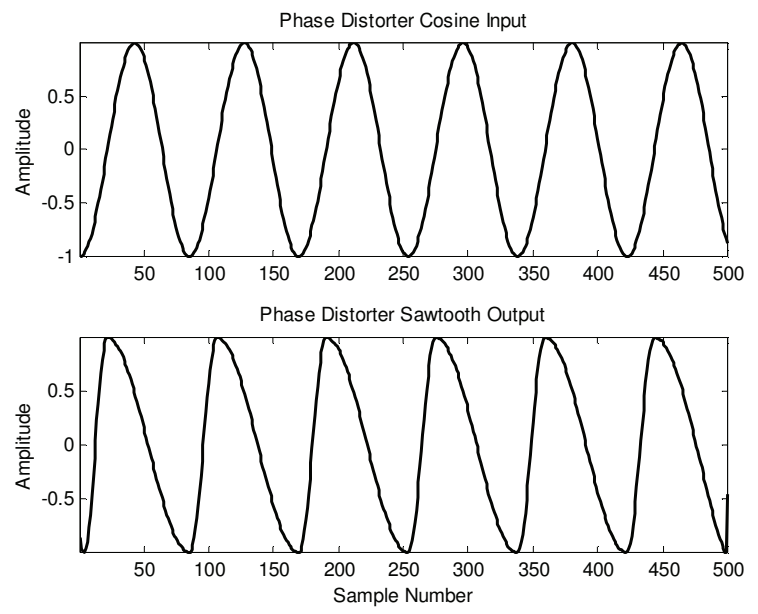

Fig. 2. Input cosine and output sawtooth of phase distorter

Assuming an input cosine wave to the phase distortion process, the algorithm essentially speeds up the cosine wave as its amplitude moves from -1 to 1 and then slows down the phase increments of the cosine wave for the rest of the cycle. This gives the output a fast rising edge followed by a slower falling edge within each cycle, thus retaining the same periodicity but with an altered shape. Figure 2 shows an example of this process for a cosine of frequency $523.2 \mathrm{~Hz}$. The cosine input is given in the upper panel and the Phase Distortion output is given in the lower panel. 
The phase distortion function in this case is a non-linear mapping function as shown by the solid line in Figure 3. It is possible to decompose this function into two parts, also shown in Figure 3: the original phase increment function for the cosine, given by the dash dotted line, and a modulating function, given by the dotted line. This modulating signal can be described by

$$
\varphi_{\text {mod }}(t)=(1-2 P) \pi(\operatorname{saw}(t, P)+1) / 2
$$

where $P \in(0,1)$ is the fraction of the period during which the sawtooth, given by $s a w(t, P)$, is increasing. Notice that in eqn. (7) the function is scaled and shifted so that it lies between 0 and $(1-2 P) \pi$. In both Figures 2 and 3 the value of $P$ is 0.25 .

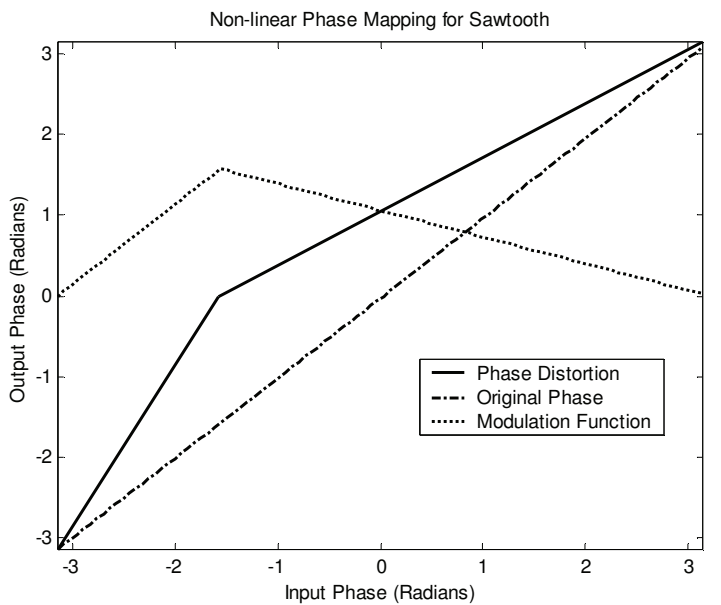

Fig. 3. Phase distortion function to generate a sawtooth

\section{PHASE DISTORTION WITH ALLPASS COEFFICIENT MODULATION}

Using eqn. (6) it should be possible to emulate the Phase Distortion technique by allpass filter coefficient modulation. However, traditional Phase Distortion is different in that it was defined to be applied on a cyclical basis to the input signal while, in contrast, the allpass coefficient modulation is not. This should result in a difference between the outputs of both techniques. To apply the modulation function defined in eqn. (7) to eqn. (6) some preliminary processing must be first carried out. While the function in eqn. (7) lies between 0 and $(1-2 P) \pi$, the phase deviation for the allpass filter lies between $-\omega$ and $-\pi$. Thus, eqn. (7) must be shifted and scaled appropriately

$$
\phi(\omega, t)=\frac{\varphi_{\text {mod }}(t)((1-2 P) \pi-\omega)}{(1-2 P) \pi}-(1-2 P) \pi-\omega
$$

Figure 4 shows the coefficient modulation function obtained using eqn. (8) from this procedure with the dashed line.

As can be seen in the figure, there are a number of significant dips in the modulation waveform. These correspond to the points of maximum phase distortion. The depth of the dips can be smoothed using a function of the form [10]

$$
m^{\prime}(t)=\alpha+\tanh (m(t)-\beta)
$$

where $\alpha$ and $\beta$ are real numbers between one and two. An example of this smoothing is given by the solid line function in Figure 4 for $\alpha=1.45$ and $\beta=1.5$.

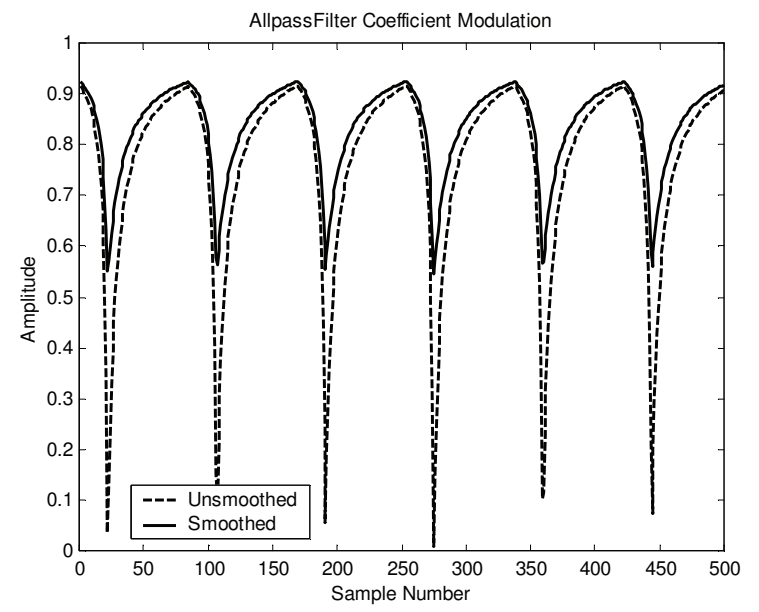

Fig.4. Coefficient modulation function from phase distortion sawtooth

To reproduce the result of Figure 3 it is also necessary to shift the phase of the allpass filter's input cosine by the same amount given in eqn. (9), that is $(1-2 P) \pi-\omega$. Applying each modulation waveform to the all pass filter for the shifted cosine input produces the results given in Figure 5.

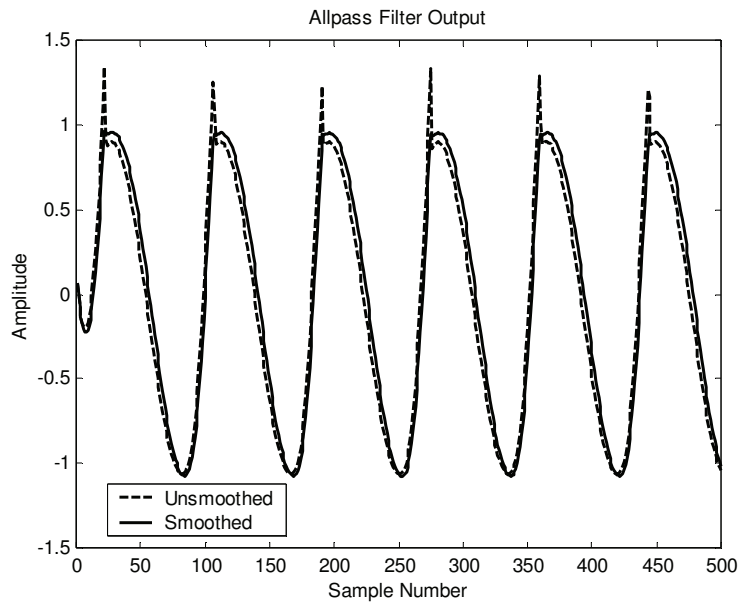

Fig. 5. Output of allpass filter with coefficient modulation

The dashed line in Figure 5 represents the output of the allpass filter for the non-smooth modulation function of Figure 4. In general, the fast rising edge followed by the slow falling edge of the phase distortion sawtooth are present in the signal. However, a significant glitch occurs at the transition between the two. In contrast the modulation 
modified by eqn. (9) provides a sawtooth without any glitches, illustrated with the solid line in Figure 5.

To illustrate the difference between the two methods for phase distortion, Figure 6 shows a plot of theoretical instantaneous frequency of the output waveforms from both techniques. For the Phase Distortion method, the first quarter period has a frequency that is twice as fast as the input. The remainder has a frequency that is two thirds as fast as the fundamental frequency. This is shown by the dashed line alternating between $1046.6 \mathrm{~Hz}$ and $348.8 \mathrm{~Hz}$ in Figure 6. The solid line in Figure 6 shows a plot of eqn. (4). Its contrasting dynamic nature is visible in the way it follows the straight dashed lines in a curving manner in keeping with the earlier assertion that it must be time-varying to introduce a frequency deviation to the signal.

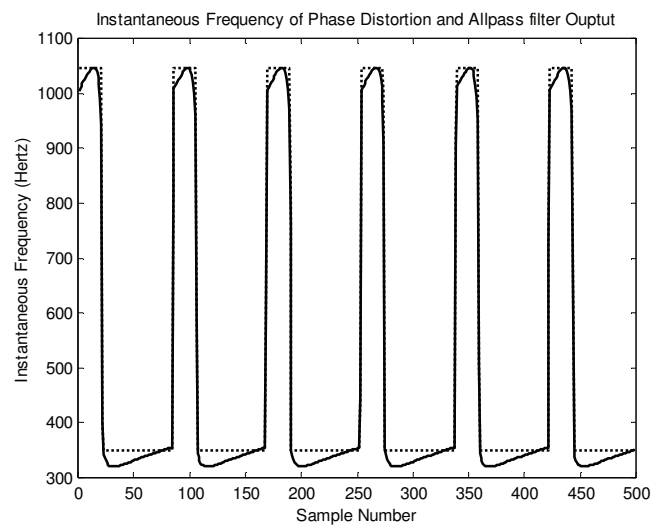

Fig. 6. The instantaneous frequency of the phase distortion sawtooth (solid line) and the allpass modulating signal (dashed line), both of fundamental frequency $532.2 \mathrm{~Hz}$

A spectral plot of the smoother sawtooth waveform shown in Figure 5 can be compared with the phase distortion sawtooth of Figure 2. These are shown in the upper and lower panels respectively of Figure 7 . It is discernable that the output of the allpass filter is richer showing a more typical spectral roll-of associated with a sawtooth wave. The spectrum of the phase distortion sawtooth is much sparser in the higher frequencies and actually has missing components, as previously noted in [1]. Some aliasing is present in both spectra, but in both cases is at very high frequencies and below $-60 \mathrm{~dB}$, rendering its perceptual effect negligible. Thus, the allpass filter method for phase distortion appears superior for producing harmonically rich spectra.

\section{CONCLUSION}

This paper has provided a novel implementation for Phase distortion sound synthesis using the allpass coefficient modulation method. Experimental results were presented to demonstrate its relationship to the standard wavetable method of Phase Distortion. The allpass filter method was shown by example to generate a spectrally richer output. Furthermore, it opens up the possibilities for novel sound processing applications by the use of arbitrary input signals in place of sinusoids.

Future work will seek to explore further this new sound processing approach. Particularly, we will investigate how it can be extended to create a variety of real-time effects for arbitrary inputs as in AdFM [3].
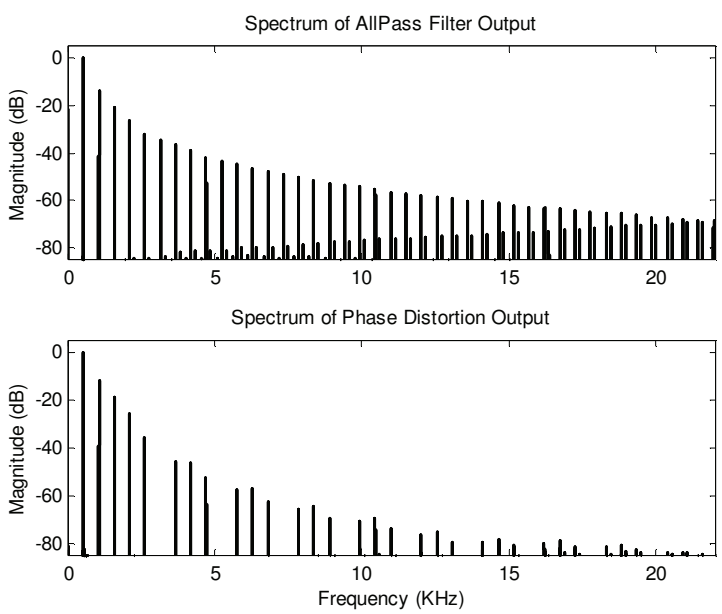

Fig. 7. Spectral plots of sawtooth output from coefficient modulated allpass filter and phase distortion

\section{ACKNOWLEDGEMENTS}

This work has been partly funded by the Academy of Finland (projects no. 122815 and 126310).

\section{REFERENCES}

[1] M. Ishibashi, Electronic musical instrument, United States Patent 4658691, filed Oct. 1985 .

[2] J. Chowning, "The synthesis of complex audio spectra by means of frequency modulations", Jnl. Audio Eng. Soc., Vol. 21, no. 7, Sept. 1973, pp. 525-534.

[3] V. Lazzarini, J. Timoney and T. Lysaght, "The generation of naturalsynthetic spectra by means of adaptive frequency modulation", Computer Music Journal, vol. 32, no. 2, summer 2008, pp. 9-22.

[4] V. Lazzarini, J. Timoney and T. Lysaght, "Non-linear distortion synthesis using the split sideband method with applications to adaptive signal processing", Jnl. Audio Eng. Soc, vol. 56, no. 9, Sept. 2008.

[5] J. Pekonen, "Coefficient Modulated first-order allpass filter as a distortion effect,", Proc. $11^{\text {th }}$ conf. Digital Audio Effects (DAFx), Espoo, Finland, Sept. 1-4, 2008.

[6] V. Lazzarini, J. Timoney and T. Lysaght, "A Modified FM synthesis approach to bandlimited signal generation", Proc. $11^{\text {th }}$ conf. Digital Audio Effects (DAFx), Espoo, Finland, Sept. 1-4, 2008.

[7] V. Lazzarini, J. Timoney and T. Lysaght, "Asymmetric-spectra methods for adaptive FM synthesis", Proc. $11^{\text {th }}$ conf. Digital Audio Effects (DAFx), Espoo, Finland, Sept. 1-4, 2008.

[8] T. I. Laakso, V. Välimäki, M. Karjalainen, and U. Laine, "Splitting the unit delay-Tools for fractional delay filter design," IEEE Sig. Proc. Mag., vol. 13, no. 1, pp. 30-60, Jan. 1996.

[9] K. Steiglitz, A digital signal processing primer, Prentice Hall, USA, Jan. 1996.

[10] R. Rojás, Neural Networks: a systematic introduction, SpringerVerlag, Berlin, 1996. 